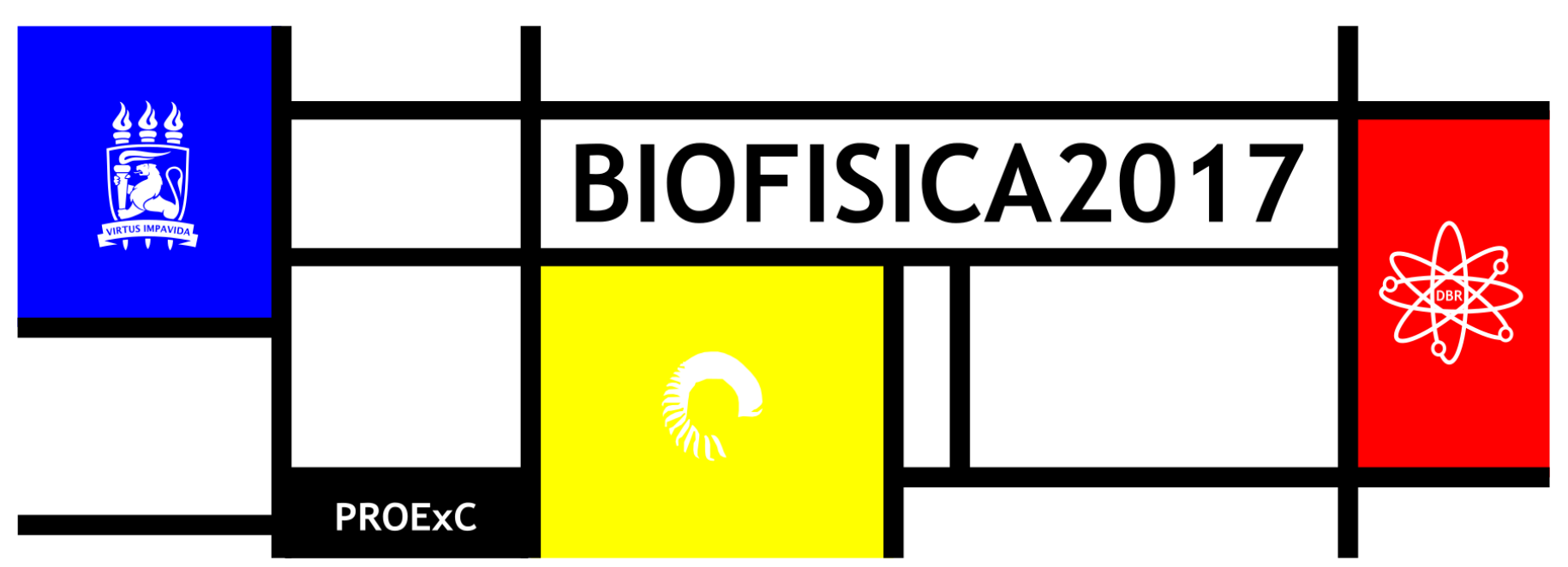

\title{
MODELO MATEMÁTICO PARA CALIBRAÇÃO DE SISTEMAS DE DETECÇÃO DE RADÔNIO-222
}

\author{
Karolayne Esthefany Melo da Silva ${ }^{1 *}$, Claudemir Batista da Silva Júnior ${ }^{1}$, Mariana Luiza de Oliveira Santos ${ }^{1}$, \\ Elvis Joacir De França ${ }^{1}$, Emerson Emiliano Gualberto de Farias ${ }^{1}$
}

${ }^{1}$ Centro Regional de Ciências Nucleares do Nordeste

*keesthefany@gmail.com

\section{INTRODUÇÃO}

O radônio-222 $\left({ }^{222} \mathrm{Rn}\right)$ é um gás incolor nas condições normais de temperatura e pressão, proveniente do decaimento do rádio-226 $\left({ }^{226} \mathrm{Ra}\right)$ da série radioativa natural do urânio-238 $\left({ }^{238} \mathrm{U}\right) .0{ }^{222} \mathrm{Rn}$ é um gás naturalmente radioativo e decai por emissão alfa. Quando inalado, pode depositar toda a energia das partículas emitidas nos pulmões, resultando em um possível desenvolvimento de câncer. Além disso, o ${ }^{222} \mathrm{Rn}$ possui radionuclídeos-filhos também emissores de partículas alfa e com energia suficiente para gerar danos aos tecidos.

Para a medição de ${ }^{222} \mathrm{Rn}$, com adequado nível metrológico, é indispensável o uso de equipamentos calibrados. Assim, o presente trabalho objetivou a avaliação de um modelo matemático para a calibração e verificação de sistema de detecção de ${ }^{222} \mathrm{Rn}$ no Centro regional de Ciências Nucleares do Nordeste - CRCN-NE.

\section{MATERIAL E MÉTODOS}

\section{AMBIENTE CALIBRAÇÃO DE DETECTORES DE ${ }^{222} \mathrm{Rn}$}

O modelo de predição leva em consideração um ambiente para calibração dos detectores denominada de Câmara de Calibração. No CRCN-NE, foi construída uma câmara e a essa foi dada uma denominação de RN1-CRCN (Figura 1).

A construção da RN1-CRCN foi baseada nos estudos desenvolvidos por Shweikani e Raja (2005). A câmara possui dimensão externa de $100 \mathrm{~cm}$ de comprimento, $85 \mathrm{~cm}$ de largura e $100 \mathrm{~cm}$ de altura, resultando em volume útil de $814,42 \mathrm{~L} \pm 0,76 \mathrm{~L}$, dispondo de uma válvula de entrada do gás gerado por uma fonte de ${ }^{226} \mathrm{Ra}$ e outra de saída, ligada ao sistema de exaustão, que não permite o aumento da concentração de ${ }^{222} \mathrm{Rn}$ no laboratório (Figura 2).

Além disso, a câmara possui cinco válvulas para acoplamento de detectores do tipo Célula de Lucas e duas conexões para detectores funcionando em modulo fluxo. Nesse caso, o sistema de detecção em teste faz uma amostragem de ar do interior da câmara e realiza a determinação do radônio presente nessa amostra, e retorna o material para o interior da câmara.
Nos ensaios, foi realizada a comparação entre valores de concentração de atividade de ${ }^{222} \mathrm{Rn}$ medidos por um detector certificado e o modelo de predição para radônio no interior da câmara RN1-CRCN.

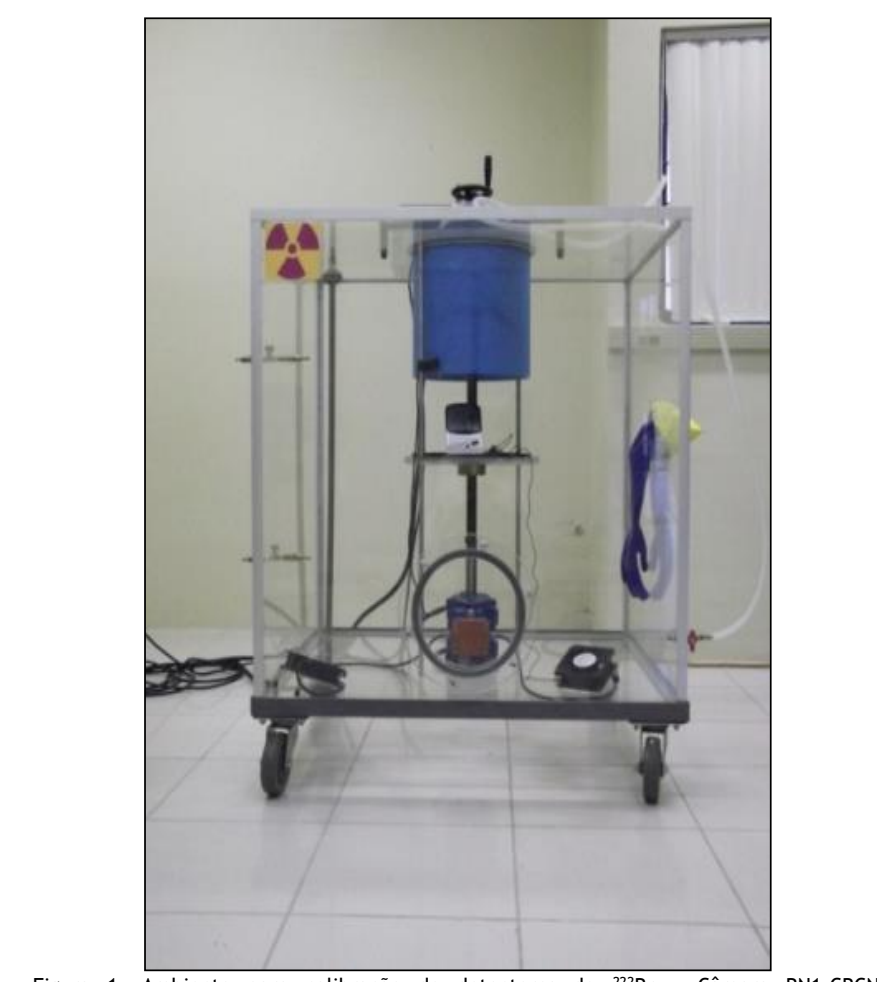

Figura 1. Ambiente para calibração de detectores de ${ }^{222} \mathrm{Rn}$ - Câmara RN1-CRCN (Farias, 2016).

Para isso, utilizou-se, além da RN1-CRCN, de fonte de ${ }^{226} \mathrm{Ra}\left(\mathrm{t}_{1 / 2}=1.600\right.$ anos) com atividade de $105 \mathrm{kBq}$, modelo PYLON RN-1025-100, Pylon Eletronics, Canadá, com taxa de produção constante de ${ }^{222} \mathrm{Rn}$. Durante os ensaios o gás foi arrastado da fonte 
de ${ }^{226} \mathrm{Ra}$ com um fluxo constante de ar para o interior da câmara, sendo o processo monitorado pelo detector certificado (Figura 2).

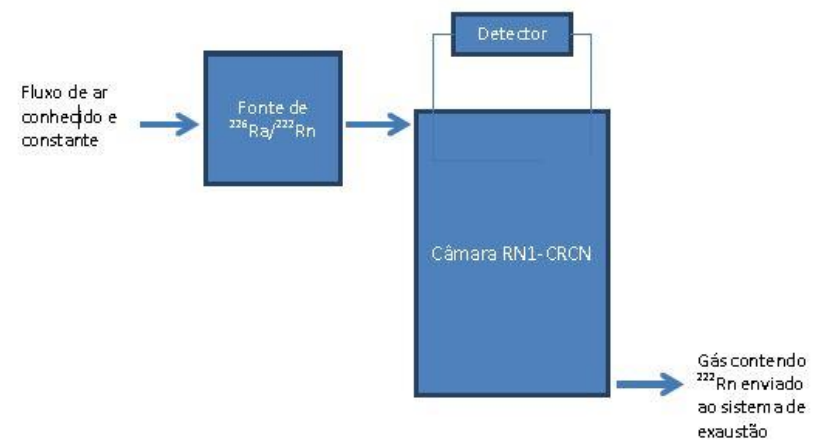

Figura 2: Arranjo experimental para a verificação do modelo teórico para calibração de detectores de radônio na câmara RN1-CRCN (Farias, 2016).

\section{MODELO DE PREDIÇÃO DE RADÔNIO NO INTERIOR DA CÂMARA}

Para a determinação da concentração teórica dentro da câmara de radônio foi utilizada a seguinte equação diferencial:

$\frac{d C R n}{d t}=\lambda_{R n} \cdot A_{R a}-\lambda_{R n} \cdot C_{R n(t)}-\frac{q 1}{V} \cdot C_{R n(t)}$

em que,

$\lambda_{R n}=$ constante de decaimento do ${ }^{222} \mathrm{Rn}$;

$A_{R a}=$ atividade da fonte de ${ }^{226} \mathrm{Ra}(105 \mathrm{KBq})$;

$C_{R n}(t)=$ concentração de ${ }^{222} \mathrm{Rn}$ no interior da câmara em função do tempo;

$q 1$ = fluxo de ar que passa através da fonte;

$V=$ Volume da câmara $(814,42$ L).

A partir da solução dessa equação diferencial foi encontrada a seguinte resolução:

$C_{R n}(t)=C_{s a t} \cdot\left(1-e^{-\lambda e \cdot t}\right)$

em que,

$C_{\text {sat }}=$ concentração de saturação de Rn na câmara;

$\lambda_{e}=$ constante efetiva modelo $\left(\lambda_{R n}+\frac{q 1}{V}\right)$;

$t=$ tempo de arraste do radônio produzido pela fonte de ${ }^{226} \mathrm{Ra}$.

Com isso, foi modelada a concentração de atividade de ${ }^{222} \mathrm{Rn}$ na condição de saturação correspondente a 99 \% e o tempo necessário para atingir essa condição, ambos os parâmetros definidos pelo fluxo de arraste.

Por meio da Equação 2, foi possível variar o fluxo de ar que atravessa a fonte de ${ }^{226} \mathrm{Ra}$ para a obtenção de concentrações de atividade em condições variadas de saturação.

Os fluxos utilizados para este ensaio foram $0,9 \mathrm{~L} \cdot \mathrm{min}^{-1}(\mathrm{~A})$; 2,2 L. $\mathrm{min}^{-1}(\mathrm{~B}) ; \quad 2,6 \mathrm{~L} \cdot \mathrm{min}^{-1}(\mathrm{C}) ; \quad 6,2 \mathrm{~L} \cdot \mathrm{min}^{-1}(\mathrm{D}) ; 8,5 \mathrm{~L} \cdot \mathrm{min}^{-1}(\mathrm{E}) \mathrm{e}$ $12 \mathrm{~L}$. min $^{-1}(\mathrm{~F})$ com as respectivas as concentrações de saturação de

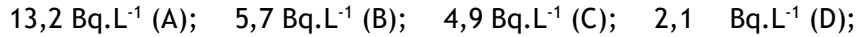
$1,5 \mathrm{~Bq} \cdot \mathrm{L}^{-1}(\mathrm{E})$ e $1,1 \mathrm{~Bq} \cdot \mathrm{L}^{-1}(\mathrm{~F})$, respectivamente.

\section{AVALIAÇÃO DO MODELO MATEMÁTICO}

O Número $E_{n}$ foi empregado para verificar a equivalência entre os resultados de concentração de atividade medidos pelo detector AlphaGUARD (Detector certificado) e o modelo teórico.

O Número $E_{n}$ avalia a qualidade dos procedimentos analíticos conforme a ISO 13528 (2005), em que valores entre -1 e 1 são indicativos de controle de qualidade do procedimento analítico em nível de $95 \%$ de confiança.

\section{RESULTADOS E DISCUSSÃO}

Os resultados da verificação do modelo teórico comparando-os com os resultados obtidos pelo detector AlphaGUARD (Detector certificado) são indicados na Tabela 1.

Tabela 1. Resultados das atividades de ${ }^{222} \mathrm{Rn}$ modeladas e certificadas da concentração de radônio na câmara de radônio, assim como o erro relativo entre as medicões. A medida de dispersão corresponde à incerteza analítica expandida em nível de $95 \%$ de confiança.

\begin{tabular}{cccc}
\hline Fluxo & $\begin{array}{c}\text { Valor } \\
\text { Modelado } \\
\left(\mathrm{kBq} \cdot \mathrm{m}^{-3}\right)\end{array}$ & $\begin{array}{c}\text { Valor } \\
\text { Certificado } \\
\left(\mathrm{kBq} \cdot \mathrm{m}^{-3}\right)\end{array}$ & $\begin{array}{c}\text { Erro } \\
\text { relativo } \\
(\%)\end{array}$ \\
\hline A & $13,2 \pm 0,7$ & $13,2 \pm 0,5$ & 0,07 \\
B & $5,7 \pm 0,4$ & $5,7 \pm 0,3$ & 0,00 \\
C & $4,9 \pm 0,3$ & $4,9 \pm 0,3$ & 0,20 \\
D & $2,10 \pm 0,10$ & $2,13 \pm 0,11$ & $-1,41$ \\
E & $1,54 \pm 0,08$ & $1,49 \pm 0,11$ & 3,15 \\
F & $1,09 \pm 0,06$ & $1,09 \pm 0,09$ & 0,64 \\
\hline
\end{tabular}

Os resultados de verificação do modelo de predição de radônio no interior da câmara proporcionaram erros relativos menores que $3,15 \%$ (Tabela 1).

Considerando-se os valores do Número $\mathrm{E}_{\mathrm{n}}$, o modelo de predição da concentração de atividade de ${ }^{222} \mathrm{Rn}$ no interior da câmara RN1. CRCN produz resultados bastante semelhantes ao valores encontrados pelo detector certificado (Figura 3).

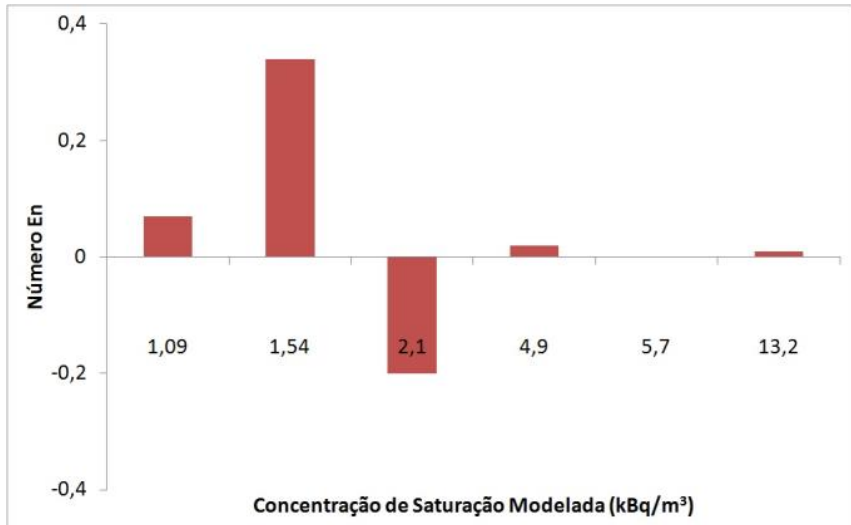

Figura 3: Resultados de Números $E_{n}$ encontrados para as diversas concentrações de saturação testadas. Valores entre -1 e 1 são indicativos de qualidade do procedimento analítico em nível de $95 \%$ de confiança

Os resultados não indicaram diferenças significativas em nível de 95\% de confiança entre os Valores Modelados e os Valores Certificados (Figura 3).

\section{CONCLUSÕES}

O modelo matemático de predição utilizado, associado a câmara de calibracão RN1-CRCN, mostrou-se como uma ótima ferramenta para a verificação e calibração de detectores de radônio-222.

\section{REFERÊNCIAS}

FARIAS, E. E. G. Exalação de Rn-222 em solos: parâmetros para modelagem e métodos de determinação. Universidade Federal de Pernambuco. Tese (Doutorado), Tecnologias Energéticas e Nucleares, p. 59-78, 2016. 
SHWEIKANI, R.; RAJA, G. Design, Construct and test of a calibration radon chamber. Radiation Measurements, v. 40, p. 316-319, 2005. 